\title{
Editorial
}

\section{Revascularização Miocárdica em Multiarteriais}

\author{
Myocardial Revascularization in Patients With Multivessel Disease
}

\author{
Alexandre A. C. Abizaid e Dimytri Alexandre Siqueira \\ São Paulo, SP
}

Os objetivos no tratamento de pacientes com doença coronariana em múltiplos vasos incluem alívio dos sintomas de isquemia, redução na taxa de eventos cardíacos (infarto, arritmias) e preservação ou restauração da função ventricular esquerda, com conseqüente maior sobrevida a longo prazo. Com a publicação de importantes estudos na década de 70, que compararam a cirurgia de revascularização miocárdica com a terapia medicamentosa ${ }^{1-3}$, entendemos que tais objetivos eram melhor alcançados com a cirurgia, especialmente quando indicada a portadores de lesão de tronco de coronária esquerda, acometimento trivascular ou disfunção ventricular.

Grandes avanços na terapêutica clínica (estatinas, antiplaquetários potentes) e cirúrgica (maior emprego de enxertos arteriais, melhor proteção miocárdica, procedimentos sem circulação extracorpórea) ocorreram desde então, com comprovada melhoria no prognóstico desses pacientes. Nos últimos anos, a intervenção coronariana percutânea tornou-se a principal forma de revascularização miocárdica empregada no tratamento da doença coronariana. $O$ incremento das técnicas e instrumentais permitiu que tal forma de revascularização, menos invasiva, pudesse ser oferecida à população de multivasculares, com segurança e eficácia semeIhantes a revascularização cirúrgica em diversos estudos. Na presente edição, Silva e cols. apresentam os resultados de estudo randomizado unicêntric ${ }^{4}$, realizado em nosso país, que avalia, a longo prazo, as vantagens das duas estratégias de revascularização sobre a evolução clínica desses pacientes.

As primeiras séries, publicadas na década de 90, compararam a cirurgia de revascularização miocárdica com angioplastia coronária por cateter-balão ${ }^{5-12}$. Esses estudos demonstraram, uniformemente, que as taxas de mortalidade e infarto não diferiam estatisticamente entre as duas formas de tratamento (fig.1); os pacientes submetidos à intervenção coronariana percutânea apresentavam, porém, maior necessidade de nova revascularização a longo prazo em razão da ocorrência de reestenose. Apesar de possuírem limitações no que se refere à seleção de pacientes (inclusão de univasculares, diferenças clínicas e angiográficas entre as populações), tais pesquisas comprovaram a segurança da angioplastia coronariana e reiteraram sua indicação a pacientes univasculares ou bivasculares com lesões menos complexas.

Instituto Dante Pazzanese de Cardiologia

Correspondência: Alexandre A. C. Abizaid - Rua Dom Paulo Pedrosa, 673/72B - 05687-001 - São Paulo - SP

E-mail: abizaid@ cardiol.br e aabizaid@uol.com.br

Enviado para publicação em 10/12/2004

202
Inicialmente utilizados para o manejo de complicações da angioplastia (oclusões agudas, dissecções do vaso), o emprego de stents coronarianos tornou-se freqüente e recomendado, em razão de suas propriedades mecânicas de impedir o recolhimento elástico e o remodelamento negativo da parede vascular - fatores determinantes no processo de reestenose coronariana após angioplastia com balão. Ao reduzir a ocorrência de reestenose e de complicações agudas, os stents aproximaram ainda mais os resultados obtidos com a intervenção coronariana percutânea e a cirurgia.

Em meta-análise ${ }^{13}$ comparativa entre a intervenção coronariana percutânea com stents e cirurgia de revascularização miocárdica, composta por 2643 pacientes, abrangendo os estudos ARTS ${ }^{14}$, ERACI II ${ }^{15}$ e SOS ${ }^{16}$, observou-se que não havia diferença nas taxas de mortalidade e infarto não-fatal entre os dois grupos ao final de 1 ano. A necessidade de nova revascularização no grupo submetido a intervenção coronariana percutânea com stents foi de 15\%, metade da incidência relatada com angioplastia por balão em multiarteriais. Os índices de nova revascularização continuavam, porém, significativamente maiores quando comparados à cirurgia de revascularização miocárdica, principalmente devido à reestenose intra-stent.

0 artigo de Silva e cols. ${ }^{4}$ corrobora tais resultados: a sobrevida livre de eventos combinados em 5 anos, no estudo em questão ( $82 \%$ para o grupo cirúrgico e $55 \%$ para o grupo ATC, $p=<0,001$ ) é semelhante à relatada no estudo ARTS I, no mesmo período de seguimento (tab.l) ${ }^{17}$.

Vivemos hoje uma nova era no tratamento percutâneo da doença arterial coronariana. 0 emprego de stents eluídos com drogas antiproliferativas, carreadas e liberadas de forma controlada na parede vascular a partir de polímeros biocompatíveis, mostrou-se efetivo na redução da hiperplasia neo-intimal, principal determinante da ocorrência de reestenose intra-stent. Desde a primeira utilização clínica de stents recobertos com sirolimus, realizada em nosso meio ${ }^{18}$, vários estudos demonstraram a segurança e eficácia desta nova tecnologia para o tratamento de lesões coronarianas de novo ${ }^{19-22}$. Em meta-análise envolvendo 5.103 pacientes ${ }^{23}$, as taxas de reestenose angiográfica e eventos cardíacos adversos (predominantemente revascularização de lesão-alvo) foram significativamente menores nos pacientes tratados com sirolimus e paclitaxel, quando comparados a stents convencionais ( $8,9 \%$ vs $29,3 \%$ e $7,8 \%$ vs $16,4 \%$, respectivamente).

Embora inicialmente aplicados a pacientes selecionados, com lesões únicas em vasos de maior calibre, os stents recobertos mostram-se eficazes também em situações mais adversas. No registro RESEARCH ${ }^{24}$, que incluiu indivíduos com lesões mais complexas (bifurcações, enxertos venosos, acometimento de tronco ou lesões longas que requeriam mais de um stent), a taxa de reestenose angiográfica evidenciada $(7,9 \%)$ foi menor que a rela- 
tada com stents não-recobertos. A observação de que o tipo de reestenose intra-stent com stents eluídos é predominante focal é de grande importância clínica, pois permite nova abordagem percutânea com menor índice de recorrência ${ }^{25}$.

0 impacto da utilização de stents eluídos com medicamentos em multiarteriais vem sendo investigado no estudo ARTS $\|^{17}$, que compara, através de análise pareada, a evolução de pacientes tratados com stents com sirolimus com a população incluída no ARTS I e submetida à cirurgia ou implante de stents não-recobertos. Resultados preliminares com seguimento de 6 meses revelam que, embora com maior percentagem de diabéticos, maior quantidade de lesões tratadas por paciente e utilização de stents mais longos em vasos mais finos (todos fatores preditores de reestenose), os indivíduos tratados com stents com sirolimus apresentam sobrevida livre de eventos maior que os pacientes avaliados no protocolo ARTS I (93,6\% vs $91 \%$ no grupo cirúrgico e $80 \%$ no grupo intervenção coronariana percutânea). A ocorrência de morte, infarto e acidente vascular encefálico foi menor no grupo sirolimus (tab. II).

Pacientes portadores de diabetes mellitus e coronariopatia multivascular apresentam pior prognóstico a curto e longo prazo quando comparados a não-diabéticos, independentemente do tipo de revascularização miocárdica empregada. A reestenose é um dos principais fatores implicados no pior prognóstico dos diabéticos submetidos à intervenção coronariana percutânea. A presença de hiperplasia neo-intimal e o maior remodelamento negativo do vaso após angioplastia, bem como o aumento de agregação plaquetária e mecanismos reduzidos de fibrinólise explicam a maior propen-

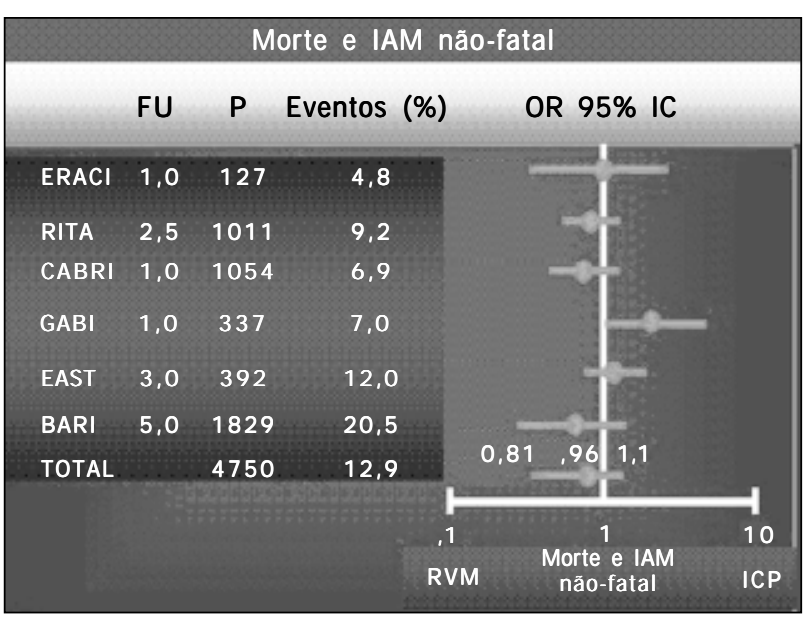

Fig. 1 - Ocorrência de morte e infarto agudo do miocárdio (IAM) não-fatal em estudos comparativos de revascularização miocárdica e intervenção coronariana percutânea ${ }^{13}$. RVM - Cirurgia de Revascularização; ICP - Intervenção Coronariana Percutânea; FU - Seguimento Follow up em anos; P - Pacientes.

\begin{tabular}{|c|c|c|c|c|}
\hline Desfecho & $\begin{array}{c}\text { RVM } \\
(n=605)\end{array}$ & $\begin{array}{c}\text { Stent } \\
(n=600)\end{array}$ & $\begin{array}{l}\text { Risco } \\
\text { relativo }\end{array}$ & $p$ \\
\hline Sobrevida (\%) & 92,4 & 92,0 & 1,05 & 0,83 \\
\hline $\begin{array}{l}\text { Sobrevida livre de } \\
\text { morte /infarto / AVC (\%) }\end{array}$ & 85,1 & 81,8 & 1,22 & 0,14 \\
\hline $\begin{array}{l}\text { Sobrevida livre de morte/ } \\
\text { infarto / AVC / nova } \\
\text { revascularização (\%) }\end{array}$ & 78,2 & 58,3 & 1,91 & $<0,001$ \\
\hline
\end{tabular}

são dos diabéticos em apresentar recorrência de obstrução coronária no sítio previamente tratado. Embora o emprego de stents tenha reduzido a incidência de reestenose, a maioria dos estudos comparativos com a cirurgia apontam para maiores taxas de nova revascularização mesmo quando utilizamos estes dispositivos $^{26}$. No estudo ARTS I, ao final do seguimento de 1 ano, os diabéticos tratados com intervenção coronariana percutânea apresentaram maior necessidade de nova revascularização quando comparados aos diabéticos submetidos à cirurgia $(22,3 \%$ vs $3,1 \%$, $p<0,001)^{27}$, ressaltando a influência da hiperplasia neointimal exagerada neste subgrupo como determinante de reestenose intrastent.

Os beneficios alcançados com a utilização de stents eluídos com medicamentos são estendidos aos diabéticos, que apresentam menor incidência de reestenose angiográfica e revascularização da lesão-alvo se tratados com stents com sirolimus, quando comparados a stents não-recobertos. Os resultados de uma subanálise do estudo SIRIUS ${ }^{28}$, que trata especificamente dos diabéticos, demonstram que a revascularização da lesão-alvo é significativamente menor quando emprega-se stents eluídos com sirolimus (22,3\% vs $6,9 \%, p<0,001)$. Quando confrontados com os resultados do estudo ARTS, percebe-se que a ocorrência de eventos cardiovasculares é significativamente menor no grupo com sirolimus (fig. 2).

As evidências de maior mortalidade a longo prazo nos diabéticos submetidos à angioplastia coronariana provém de 2 estudos $\left(C A B R I^{6}\right.$ e BARI $\left.{ }^{7}\right)$, conduzidos ainda na era pré-stents. Em subanálise (não pré-especificada) do estudo $B A R I^{29}$, os pacientes diabéticos multivasculares submetidos à cirurgia de revascularização miocárdica apresentaram, após 5 anos, mortalidade geral menor que aqueles submetidos à angioplastia com balão $(19,4 \% \times 34,5 \%$, $p=0,0024)$. Este benefício da cirurgia sobre a angioplastia foi restrito aos diabéticos revascularizados com implante de artéria torácica interna para a descendente anterior, sendo a evolução dos que receberam exclusivamente enxertos venosos semelhante aos tratados por angioplastia. Tal observação não foi corroborada por grandes registros (inclusive o próprio registro do BARI) e outras séries mais contemporâneas. No estudo ARTS ${ }^{30}$, quando se analisa o subgrupo dos diabéticos (208 pacientes), a mortalidade após 3 anos de acompanhamento foi de 7,1\% no grupo submetido a angioplastia e 4,2\% no grupo cirúrgico ( $p=N S$ ).

$\mathrm{Na}$ era dos stents eluídos com medicamentos, não há dúvidas de que melhor evolução clínica será experimentada pelos pacientes multiarteriais submetidos à intervenção percutânea, em função de revascularização mais duradoura. Contudo, importantes questões ainda devem ser analisadas antes da generalização de resultados para a prática clínica.

A maioria dos estudos em multivasculares determinaram como critério de inclusão a necessidade de se obter revascularização miocárdica completa tanto por intervenção coronariana percutânea como por cirurgia de revascularização miocárdica. Indivíduos com anatomia coronariana mais extensa e complexa, disfunção ventricular esquerda e já revascularizados previamente por intervenções percutâneas foram sistematicamente excluídos. Da mesma forma, portadores de síndromes coronarianas agudas, insuficiência renal, doença cerebrovascular constituíram minoria da população. Portanto, a população analisada foi constituída por pacientes multivasculares com perfil clínico e angiográfico de menor severidade. A melhor estratégia de revascularização em pacientes mais graves ainda não é definida. 
Revascularização Miocárdica em Multiarteriais

\begin{tabular}{|c|c|c|c|}
\hline Desfechos & $\begin{array}{c}\text { ARTS II - SES (\%) } \\
N=606\end{array}$ & $\begin{array}{c}\text { ARTS I - CRVM (\%) } \\
\text { N }=605\end{array}$ & $\begin{array}{c}\text { ARTS I - ICP (\%) } \\
N=600\end{array}$ \\
\hline Morte & 0,5 & 1,8 & 2,3 \\
\hline AVC & 0,5 & 1,2 & 1,5 \\
\hline Infarto do Miocárdio & 0,7 & 3,8 & 4,5 \\
\hline Nova CRVM & 1,6 & 0,5 & 3,8 \\
\hline Nova ICP & 3,1 & 2,0 & 7,8 \\
\hline Eventos combinados & 6,4 & 9,0 & 20 \\
\hline
\end{tabular}

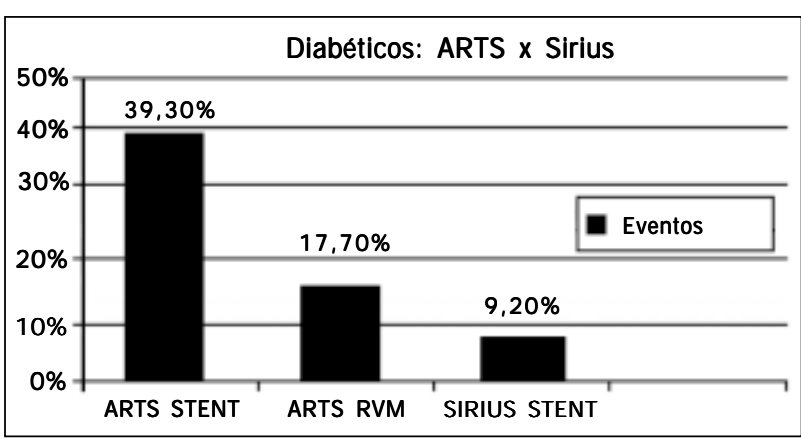

Fig. 2 - Eventos cardiovasculares (Morte, infarto agudo do miocárdio e revascularização de vaso-alvo) em 12 meses em diabéticos incluídos nos estudos ARTS e SIRIUS.

0 estudo SINTAX ${ }^{17}$ pretende randomizar 1.500 portadores de doença de tronco da coronária esquerda ou acometimento multivascular para intervenção percutânea com implante de stents eluídos com paclitaxel e cirurgia de revascularização. A inclusão de indivíduos com perfil clínico mais grave (presença de disfunção ventricular, insuficiência renal e acidente vascular encefálico prévio), bem como portadores de lesões complexas (oclusões crônicas, por exemplo) aproximará a população estudada daquela do mundo real

Embora não uniforme, a observação de maior mortalidade a longo prazo nos diabéticos submetidos à angioplastia coronariana ainda é motivo de debate. 0 estudo FREEDOM ${ }^{17}$, multicêntrico e randomizado, pretende avaliar a evolução de 2.400 diabéticos submetidos à cirurgia de revascularização miocárdica (com ou sem circulação extracorpórea) e intervenção coronariana com uso de stents com sirolimus e paclitaxel. Com seguimento clínico de 3 anos, a comparação de ocorrência de desfechos primários (morte, infarto e acidente vascular encefálico) fornecerá informações relevantes para o manejo deste subgrupo de pacientes.

$\mathrm{Na}$ abordagem de pacientes com doença coronária multivascular, os limites definidores da estratégia a ser empregada têm se tornado menos rigorosos. A apresentação clínica, extensão e severidade da doença coronária, a presença de disfunção ventricular esquerda e comorbidades continuam a influenciar a escolha do tratamento inicial. $\mathrm{Na}$ era dos stents eluídos com medicamentos, vislumbra-se que a indicação de intervenção coronariana percutânea será ampliada, uma vez que reestenose não será a principal limitação. Neste contexto, a redução de eventos cardíacos (morte, infarto) se estabelece como o objetivo primário a ser alcançado. Além de tratamento medicamentoso eficaz, o desenvolvimento e aprimoramento de métodos para a identificação de indivíduos e lesões coronarianas vulneráveis e a avaliação da terapêutica recomendada (inclusive abordagem de lesões propensas à instabilização com stents recobertos) serão requeridos.

\section{Referências}

1. European Coronary Surgery Study Group. Long-term results of prospective randomized study of coronary artery bypass surgery in stable angina pectoris. Lancet 1982;2:1173-80.

2. Alderman EL, Bourassa MG, Cohen LS, et al. Ten-year follow-up of survival and myocardial infarction in the randomized Coronary Artery Surgery Study. Circulation 1990;82:1629-46.

3. Detre KM, Takaro T, Hultgren $\mathrm{H}$, et al. Long-term mortality and morbidity results of the Veterans Administration randomized trial of coronary artery bypass surgery. Circulation 1985;72(supl 5): 84

4. Silva PRD, Hueb WA, César LAM, Oliveira SA, Ramires J AF. Estudo comparativo dos resultados da intervenção cirúrgica e da angioplastia na revascularização do miocárdio em portadores de comprometimento multiarterial equivalente. Arq Bras Cardiol 2005;84:214-21

5. RITA Trial Participants. Coronary angioplasty vs coronary artery bypass surgery: the Randomized Intervention Treatment of Angina (RITA) trial. Lancet 1993;343:573-80.

6. CABRI Trial Participants. First-year results of CABRI (Coronary Angioplasty vs. Bypass Revascularization Investigation). Lancet 1995:346: 1179-84.

7. The Bypass Angioplasty Revascularization (BARI) Investigators. Comparision of coronary bypass surgery with angioplasty in patients with multivessel disease. $\mathrm{N}$ Engl] Med 1996;335:217-25.

8. Rodriguez A, Boullon F, Perez-Balino N, et al. Argentine randomized trial of percutaneous transluminal coronary angioplasty vs coronary artery bypass surgery in multivessel dissease (ERACl): in-hospital results and 1-year follow-up. J Am Coll Cardiol 1993;22: 1060-7. gery Investigation. A randomized study of coronary angioplasty compared with bypass surgery in patients with symptomatic multivessel coronary disease. $\mathrm{N}$ Engl J Med 1994;331: 1037-43.

10. King SB, Lembo NJ, Weintraub WS, et al. A randomized trial comparing coronary angioplasty with coronary bypass surgery. N Engl J Med 1994;331: 1044-50.

11. BARI investigators. Influence of diabetics on 5 -year mortality and morbidity in a randomized trial comparing CABG and PTCA in patients with multivessel disease. Circulation 1997:96: 1761-9.

12. King SB, Kosinski AS, Guyton RA, et al. Eight-year mortality in the Emory Angioplasty vs Surgery Trial (EAST). J Am Coll Cardiol 2000;35: 1116 -21.

13. Hoffman SN, TenBrook J A, WolfWP, et al. A meta-analysis of randomized controlled trials comparing coronary bypass graft with percutaneous transluminal coronary angioplasty: one to eight-year outcomes. J Am Coll Cardiol. 2003;41: 1293-304.

14. Serruys PW, Unger F, Sousa JE, et al. Comparison of coronary artery bypass surgery and stenting for the treatment of multivessel disease. N Engl J Med 2001;344: 1117-24.

15. Rodriguez A, Bernardi V, Navia J , et al. Argentine randomized study: coronary angioplasty with stenting vs coronary bypass surgery in patients with multiple-vessel disease (ERACI-II): 30 day and one-year follow-up results. J Am Coll Cardiol 2001;37:51-8.

16. SoS Investigators. Coronary artery bypass vs percutaneous coronary intervention with stent implantation in patients with multivessel coronary artery disease (the Stent or Surgery trial): a randomized controlled trial. Lancet 2002;360: 965-70.

17. Disponível na internet: http. www. theheart. org e tctmd.com.

18. Sousa JE, Costa MA, Abizaid A, et al. Lack of neointimal proliferation after im- 
plantation of sirolimus-coated stents in human coronary arteries: a quantitative coronary angiography and three-dimensional ultrasound study. Circulation 2001; 103: 192-95

19. M orice MC, Serruys PW, Sousa JE, et al. A randomized comparision of a sirolimuseluting stent with a standard stent for coronary revascularization. $\mathrm{N}$ Engl J Med 2002;346: 1773-80.

20. Moses J W, Leon MB, Popma J J, et al. Sirolimus-eluting stents vs standard stents in patients with stenosis in a native coronary artery. N Engl J Med 2003;349: 1315-23.

21. Stone GW, Ellis SG, CoxDA, et al. A polymer-based, paclitaxel-eluting stent in patients with coronary artery disease. N EnglJ Med 2004;350: 221-31.

22. Park SJ , Shin WH, Ho DS, et al. A paclitaxel-eluting stent for the prevention of Coronary restenosis. N EnglJ Med. 2003;348: 1537-45.

23. Babapulle $M N$, J oseph $L$, Belisle $P$, et al. A hierarchical bayesian meta-analysis of randomized clinical trials of drug-eluting stents. Lancet 2004;364: 557-8.

24. Lemos PA, Hoye A, Goedhart D, et al. Clinical, angiographic and procedural predictors of angiographic restenosis after sirolimus-eluting stent implantation on complex patients. Circulation 2004;109: 1366-70.
25. Lemos PA, Saia F, Lighart J M, et al. Coronary restenosis after sirolimus-eluting stent implantation: morphological description and mechanistic analysis from a consecutive series of cases. Circulation 2003;108: 257-60.

26. Abizaid A, Kornowsky R, Mintz GS, et al. The influence of diabetes mellitus on acute and late clinical outcomes following coronary stent implantation. I Am Coll Cardiol 1998;32: 584-9.

27. Abizaid A, Costa MA, Centemero M, et al. Clinical and economic impact of diabetes mellitus on percutaneous and surgical treatment of multivessel coronary artery disease patients: insights from the Arterial Revascularization Therapy Study (ARTS) trial. Circulation 2001;104: 533-8.

28. Moussa I, Leon MB, Baim DS, et al. Impact of sirolimus-eluting stents on outcome in diabetic patients. Circulation 2004;109: 2273-8.

29. Detre K, Guo P, Holubkov R, et al. Coronary revascularization in diabetic patients: a comparison of the randomized and observational components of the Bypass Angioplasty Revascularization Investigation (BARI). Circulation 1999;99: 633-40

30. Legrand VMG, Serruys PW, Unger F, et al. Three-year outcome after coronary stenting vs bypass surgery for the treatment of multivessel disease. Circulation 2004; 109: 1114-20.

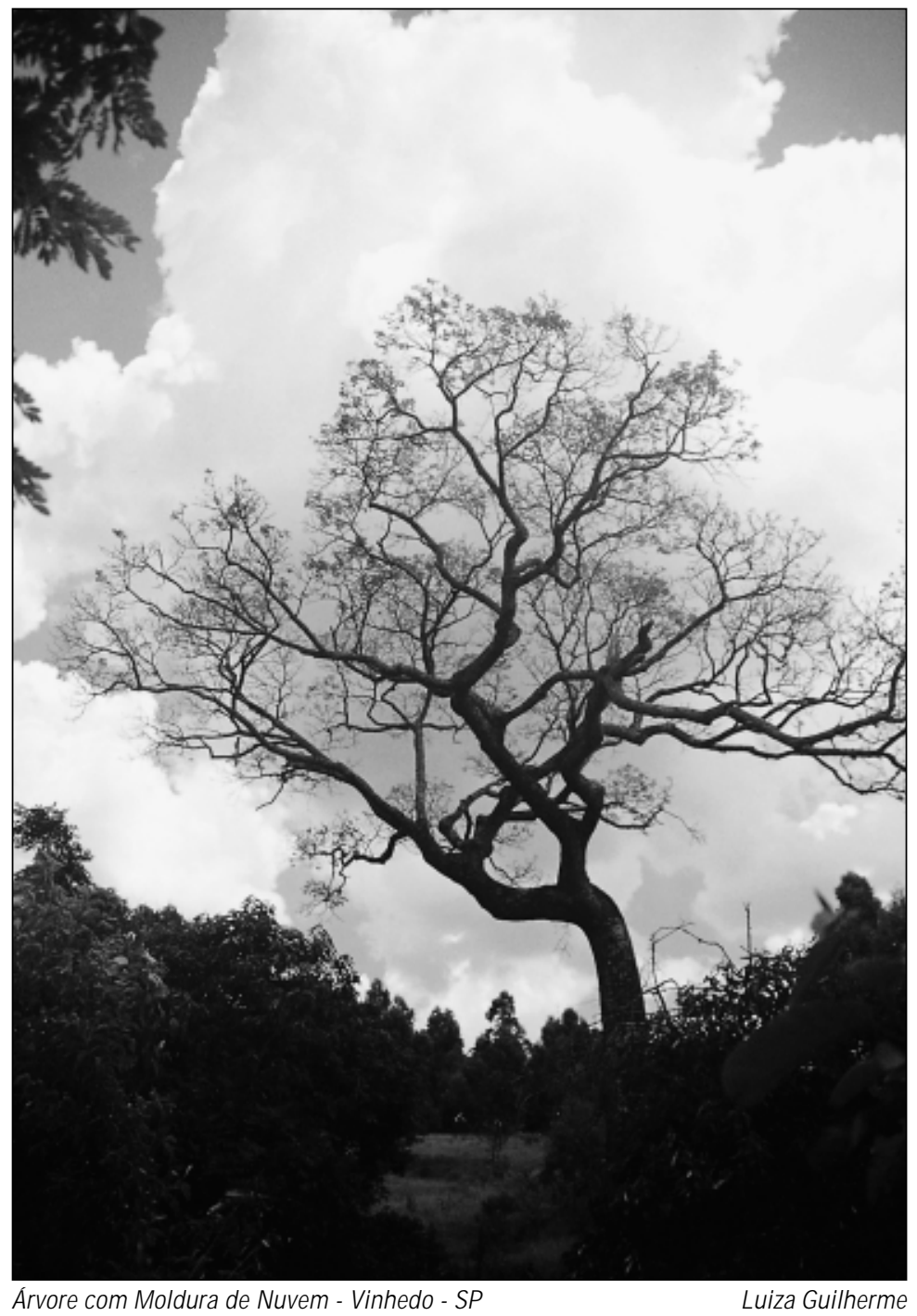

Editor da Seção de Fotografias Artísticas: Cícero Piva de Albuquerque

Correspondência: InCor - Av. Dr. Enéas C. Aguiar, 44 - 05403-000 - São Paulo, SP - E-mail: delcicero@ incor. usp.br 